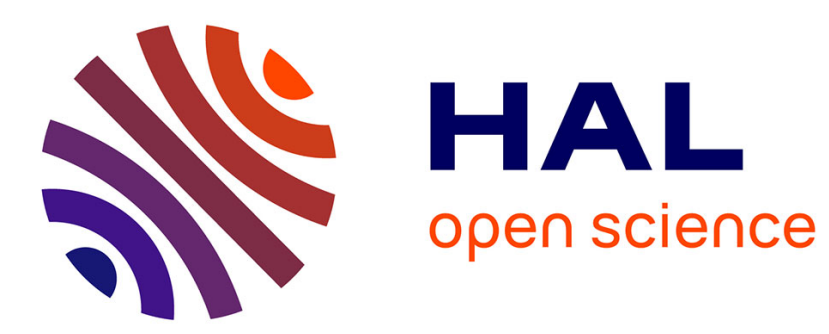

\title{
A hybrid predictive control algorithm for tracking in a single-phase DC/AC inverter
}

Luca Torquati, Ricardo Sanfelice, Luca Zaccarian

\section{To cite this version:}

Luca Torquati, Ricardo Sanfelice, Luca Zaccarian. A hybrid predictive control algorithm for tracking in a single-phase DC/AC inverter. 2017 IEEE Conference on Control Technology and Applications (CCTA), IEEE, Aug 2017, Mauna Lani Resort, United States. 10.1109/CCTA.2017.8062574 . hal01700154

\section{HAL Id: hal-01700154 \\ https://hal.laas.fr/hal-01700154}

Submitted on 17 Feb 2018

HAL is a multi-disciplinary open access archive for the deposit and dissemination of scientific research documents, whether they are published or not. The documents may come from teaching and research institutions in France or abroad, or from public or private research centers.
L'archive ouverte pluridisciplinaire HAL, est destinée au dépôt et à la diffusion de documents scientifiques de niveau recherche, publiés ou non, émanant des établissements d'enseignement et de recherche français ou étrangers, des laboratoires publics ou privés. 


\title{
A Hybrid Predictive Control Algorithm for Tracking in a Single-Phase DC/AC Inverter
}

\author{
Luca Torquati ${ }^{1}$, Ricardo G. Sanfelice ${ }^{2}$, and Luca Zaccarian $^{3}$
}

\begin{abstract}
In this paper we design a hybrid predictive controller for the tracking of a sinusoidal reference signal. The stability and forward invariance of a set of points around the reference state, named the tracking ellipse, is established by using tools for hybrid dynamical systems. Moreover, prediction of solutions for a finite number of switching events is used to minimize the number of switches. The control algorithm is shown to be robust to small perturbations and input disturbances. Simulations illustrating the main results are included.
\end{abstract}

\section{INTRODUCTION}

The future of power generation and distribution is strongly and inevitably connected to the idea of smart grid. This concept is based on a two-way flow of electricity and information, and on the capability of monitoring power plants, individual appliances and customer preferences. It incorporates into the grid the benefits of distributed computing and communications to deliver real-time information and enable the nearinstantaneous balance of supply and demand at the device level. In such a system, an essential role is played by the interconnection between appliances, energy storage units and renewable energy sources, such as photo-voltaic arrays, solar panels, wind turbines and hydroelectric plants. In such an interconnection, the DC/AC power conversion stage is critical when taking into account the high dependence of the power generation process on environmental conditions, as in the case of renewable energy sources.

\footnotetext{
${ }^{1}$ Dipartimento di Ingegneria Industriale, University of Trento, Italy, luca.torquati@unitn.it

${ }^{2}$ Department of Computer Engineering, University of California, Santa Cruz, CA, USA.ricardo@ucs c . edu. The work of R.G. Sanfelice has been partially supported by the National Science Foundation under CAREER Grant no. ECS-1450484 and Grant no. CNS-1544396, and by the Air Force Office of Scientific Research under Grant no. FA9550-16-1-0015.

${ }^{3}$ CNRS, LAAS, 7 avenue du Colonel Roche, F-31400 Toulouse, France and Universite de Toulouse, 7 avenue du Colonel Roche, 31077 Toulouse cedex 4, France, and Dipartimento di Ingegneria Industriale, University of Trento, Italy zaccariand laas.fr
}

This paper focuses on the analysis of a single-phase inverter, the most widely used device for DC/AC power conversion. The vast majority of modern controllers for DC/AC inverters implement algorithms designed using Pulse Width Modulation (PWM) techniques [1], [2]. The basic idea in most PWM-based algorithms for the control of the H-bridge in a DC/AC converter is to compare a sinusoidal carrier signal at the desired frequency to a triangular waveform. The frequency of the triangular waveform establishes the inverter switching frequency and, along with its amplitude, is generally kept constant [1]. An input voltage regulator is typically used to provide constant amplitude and overcome the lack of robustness of the PWM scheme to DC input perturbations [3]. Another drawback of this approach is the high harmonic distortion it produces, which is an important limitation when considering the increasing demand of output power quality, especially in the case of grid-tied inverters.

In this paper, we present a hybrid predictive controller for the tracking of a sinusoidal reference signal in a single-phase DC/AC inverter. The proposed control strategy includes an algorithm that guarantees uniform local asymptotic stability of a tunable (in size) set of points described by an ellipse centered at the reference state. This local asymptotic stability property is enforced by selecting one among a finite set of admissible switching configurations of the $\mathrm{H}$-bridge in the DC/AC inverter. To optimize such a selection, we propose a prediction-based algorithm that maximizes the time between switches. Robustness to small perturbations, input voltage disturbances and impulsive resets of the reference signal is shown numerically, along with frequency domain analysis of the output signal which reveals that the total harmonic distortion complies with IEEE standard specifications for grid-tied inverters.

The remainder of this paper is organized as follows. Section III illustrates the model describing the dynamics of the single-phase DC/AC inverter. Detailed analysis of the reference dynamics and the control algorithm are provided in Section III. Finally, Section IV 
illustrates the main results obtained with the proposed controller. Proofs of the results and some details are omitted due to space limitations, and will be published elsewhere.

\section{Analytical Model of a Single-Phase DC/AC INVERTER}

A single-phase DC/AC inverter consists of four controlled switches forming an H-bridge and a series RLC filter, as shown in Figure 1. The purpose of this circuit is to convert a DC input signal $V_{D C}$ in order to obtain an approximation of a sinusoidal voltage $v_{C}$ across the output capacitor $C$. The current across the inductor $L$ is denoted as $i_{L}$. The control action consists in toggling switches $S_{1}$ to $S_{4}$ according to a specific logic. In this model, the switches are assumed to be ideal, therefore the "ON" and "OFF" stages correspond to closed and open circuit connections, respectively. Moreover, we consider a negligible switching time. Depending on the position of the switches, three different configurations are possible for the voltage $V_{i n}$ to the RLC filter: $V_{D C}$, $-V_{D C}$ or 0 . These combinations lead to the following dynamics of the circuit in Figure 1 .

$\dot{i}_{L}=\left\{\begin{array}{rr}\frac{V_{D C}}{L}-\frac{R}{L} i_{L}-\frac{1}{L} v_{C} & \begin{array}{r}\text { when } S_{1}=S_{3}=\mathrm{ON} \\ \text { and } S_{2}=S_{4}=\mathrm{OFF}\end{array} \\ -\frac{V_{D C}}{L}-\frac{R}{L} i_{L}-\frac{1}{L} v_{C} & \begin{array}{r}\text { when } S_{1}=S_{3}=\mathrm{OFF} \\ \text { and } S_{2}=S_{4}=\mathrm{ON}\end{array} \\ -\frac{R}{L} i_{L}-\frac{1}{L} v_{C} & \begin{array}{r}\text { when } S_{1}=S_{4}=\mathrm{OFF} \\ \text { and } S_{2}=S_{3}=\mathrm{ON} ;\end{array}\end{array}\right.$

$\dot{v}_{C}=\frac{1}{C} i_{L}$.

We denote the configuration of the H-bridge by $q \in$ $Q:=\{-1,0,1\}$, where $q=-1$ corresponds to $V_{i n}=$ $-V_{D C}, q=0$ to $V_{i n}=0$, and $q=1$ to $V_{i n}=V_{D C}$. Then, we may model the system as in [4]:

$$
\dot{z}=\left[\begin{array}{c}
\dot{i}_{L} \\
\dot{v}_{C}
\end{array}\right]=f_{z}(q, z):=\left[\begin{array}{c}
\frac{V_{D C}}{L} q-\frac{R}{L} i_{L}-\frac{1}{L} v_{C} \\
\frac{1}{C} i_{L}
\end{array}\right],
$$

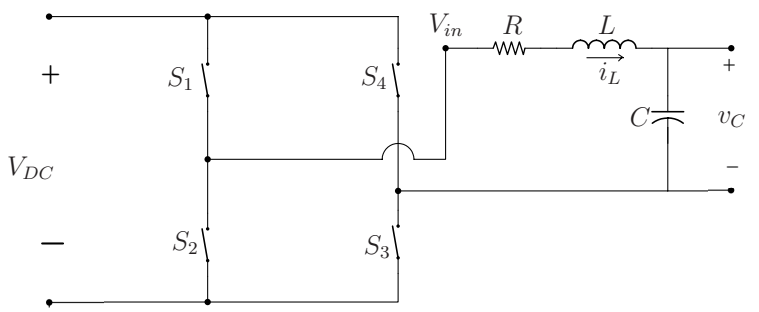

Fig. 1: Single-phase DC/AC inverter circuit diagram. where $V_{D C}$ is the input DC voltage and $R, L, C$ are parameters of the circuit. As mentioned in Section I] a PWM-based controller switches among some of these configurations depending on the difference between a reference carrier and a modulating signal. On the other hand, the proposed hybrid controller selects the configuration of the switches using the measured values of $i_{L}, v_{C}$, the reference state to be tracked, and the current switch configuration $q$.

\section{Hybrid PREdictive Control For the TraCKING OF A SinUSOIDAL Voltage}

In this section, a hybrid predictive control strategy for tracking of a reference trajectory for a singlephase DC/AC inverter is developed. This approach is inspired by the work in [4] and provides an alternative to traditional PWM-based controls. The general idea behind the proposed approach is to generate an AC voltage at the output of the inverter circuit by tracking a sinusoidal reference signal. This task is accomplished by guaranteeing that the state of the inverter converges to a tracking ellipse centered at the moving reference state.

The system architecture is depicted in Figure 2. The hybrid controller $\mathcal{H}_{c}$ locally controls the state $z$ so as to remain inside a given neighborhood of the reference state $z_{r}$, which we will refer to as "tracking ellipse". In the following section, we introduce the reference dynamics and the algorithm implemented by the controller. The properties of the resulting closedloop system are then analyzed.

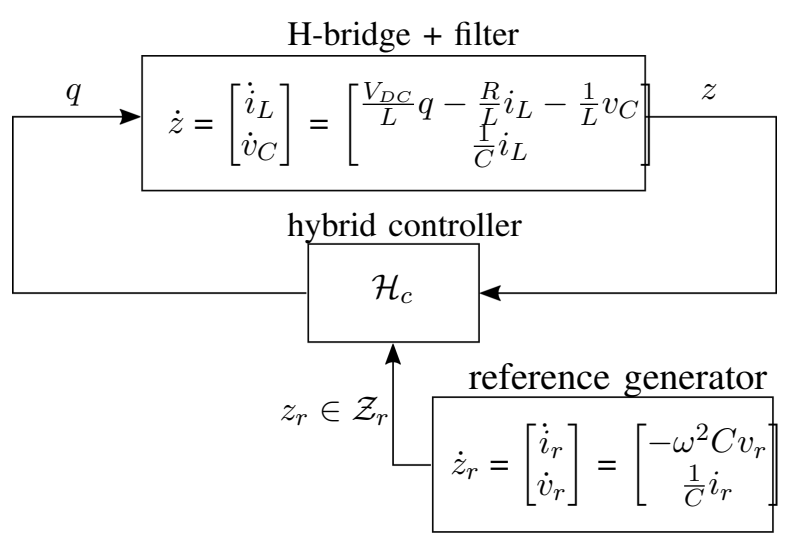

Fig. 2: Block diagram of the closed-loop system.

The closed-loop system dynamics can be described by the following states:

- $z:=\left(i_{L}, v_{C}\right) \in \mathbb{R}^{2}$ is the state of the circuit with dynamics defined in (2); 
- $z_{r}:=\left(i_{r}, v_{r}\right) \in \mathbb{R}^{2}$ is the reference state;

- $q \in Q$ is a logic variable denoting the configuration of the H-bridge switches.

\section{A. Reference Trajectory}

The objective of the proposed hybrid predictive controller is to track a sinusoidal reference signal $t \mapsto$ $z_{r}(t)$. The reference dynamics can be computed by first considering the desired output voltage as $v_{r}(t)=$ $A \sin (\omega t+\theta)$, where $A$ and $\omega$ are the desired amplitude and frequency for the output AC voltage, respectively, and $\theta$ is the initial phase angle. By using Kirchoff's laws for the circuit in Figure 1, the reference trajectory $t \mapsto z_{r}(t)$ can be seen as the unique solution to the initial value problem:

$$
\dot{z}_{r}=f_{z_{r}}\left(z_{r}\right):=\left[\begin{array}{c}
-\omega^{2} C v_{r} \\
\frac{1}{C} i_{r}
\end{array}\right], \quad z_{r}(0)=\left[\begin{array}{c}
\omega C A \cos \theta \\
A \sin \theta
\end{array}\right],
$$

where $f_{z_{r}}$ defines a linear oscillator. On the phase plane $\left(i_{r}, v_{r}\right)$, the reference trajectory describes an ellipse with axes $a=\omega C A$ and $b=A$, and aspect ratio $\frac{a}{b}=$ $\omega C$, as depicted in Figure 3. In a practical application scenario, the reference signal can either be internally generated by the controller or, more generally, received from an external source. An example can be the output synchronization of a network of inverters in a microgrid, in which the combination of these signals has to be further synchronized to the grid AC voltage. In this paper we assume that $z_{r}$ evolves in the compact set

$$
\mathcal{Z}_{r}:=\left\{z_{r} \in \mathbb{R}^{2}: \frac{i_{r}^{2}}{(\omega C A)^{2}}+\frac{v_{r}^{2}}{A^{2}}=1, A \in\left[0, A_{r}\right]\right\},
$$

which includes all the admissible periodic orbits generated by the sinusoidal reference signal, where the value of the parameter $A_{r}$ will be defined in Section III-B.

\section{B. Tracking ellipse}

Consider the dynamics of the error state $e:=$ $\left(e_{i}, e_{v}\right):=\left(i_{L}-i_{r}, v_{C}-v_{r}\right) \in \mathbb{R}^{2}$ given by

$\dot{e}=\left[\begin{array}{c}\dot{e}_{i} \\ \dot{e}_{v}\end{array}\right]=\left[\begin{array}{cc}0 & -\omega^{2} C \\ \frac{1}{C} & 0\end{array}\right] e+\left[\begin{array}{c}\nu(q, z) \\ 0\end{array}\right]=: A_{e} e+b_{e}(q, z)$

where

$$
\nu(q, z):=\frac{V_{D C}}{L} q-\frac{R}{L} i_{L}+\frac{L C \omega^{2}-1}{L} v_{C} .
$$

Given the parameters $h \in \mathbb{R}_{>0}, \psi \in \mathbb{R}_{\geq 0}$, the ellipse centered at the origin $e=(0,0)$ is identified by the set of points in a level set of the function $V: \mathbb{R}^{2} \rightarrow \mathbb{R}$ given by

$$
V(e):=e^{\top}\left[\begin{array}{cc}
h & \psi / 2 \\
\psi / 2 & (C \omega)^{2}
\end{array}\right] e=e^{\top} P e .
$$

The ellipse axes are rotated with respect to the $\left(e_{i}, e_{v}\right)$ frame by an angle $\Theta \in\left[-\frac{\pi}{4}, \frac{\pi}{4}\right]$ given by (see Figure 3)

$$
\Theta=\frac{1}{2} \tan ^{-1}\left(\frac{\psi}{h-(C \omega)^{2}}\right) .
$$

Remark 1: In this paper, we will use $h=1$ and $\psi=\frac{R C}{L}$ to simplify the design of the controller. It is possible, however, to derive similar constructions for generic values of such parameters.

Let the parameter $\rho \in \mathbb{R}_{>0}$ denote the size of the tracking ellipse, whose axes in the rotated frame are determined by $a_{\rho}=\sqrt{\frac{\rho}{h}}$ and $b_{\rho}=\frac{\sqrt{\rho}}{C \omega}$ (see Figure 3 . Given scalars $h, \rho \in \mathbb{R}_{>0}$ and $\psi \in \mathbb{R}_{>0}$, the set $\Lambda(\rho)$ defines the closed tracking ellipse of size $\rho$ centered at the reference state:

$$
\Lambda(\rho):=\left\{e \in \mathbb{R}^{2}: V(e) \leq \rho\right\} .
$$

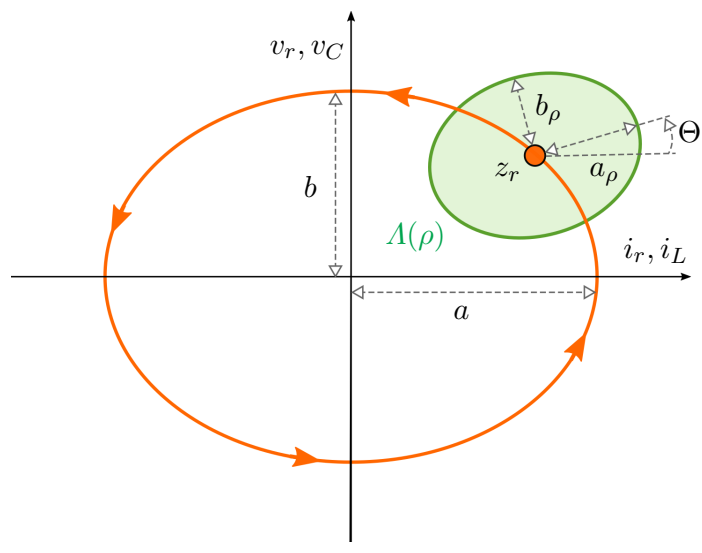

Fig. 3: Tracking ellipse $\Lambda(\rho)$ evolving along the periodic orbit $\mathcal{Z}_{r}$.

Next, we present some key properties of $V$. Let $\mathcal{X}:=$ $Q \times \mathbb{R}^{2} \times \mathcal{Z}_{r}$ and $\Gamma \subset \mathbb{R}$ be defined as

$$
\Gamma:=\left\{v_{C} \in \mathbb{R}:\left|v_{C}\right| \leq \frac{V_{D C}-\omega R C A}{k}\right\},
$$

where $k:=\left|L C \omega^{2}-1\right|$.

Lemma 1: Consider positive scalars $\rho, L, C, R, \omega, V_{D C}, \quad h=1, \quad \psi=\frac{R C}{L}$, such that $k>0$ and $R<2 \omega L$. Then for all $v_{C} \in \Gamma$ there exists at least one $q \in Q$ such that

$$
\dot{V}(e, q, z) \leq-\frac{R}{L} V(e) .
$$


When conditions satisfying Lemma 1 are met, it is always possible to select one among the values of $q \in Q$ such that function $V$ is strictly decreasing, i.e., solutions to the error dynamics (4) flow towards smaller level sets of $V$. Then, since $\Lambda(\rho)$ is a sublevel set of $V$ itself, it is possible to design a controller with the objective of rendering the tracking ellipse $\Lambda(\rho)$ uniformly locally asymptotically stable.

Let us introduce the notion of admissible tracking set, which will be used to characterize any sublevel set of $V$ in which $\dot{V}$ can be guaranteed to be strictly negative.

Definition 1: Given positive scalars $\rho, L, C, R, \omega, V_{D C}, h=1, \psi=\frac{R C}{L}$, such that $k>0$ and $R<2 \omega L$, a $\delta$-sublevel set of $V$, denoted as $\Lambda(\delta)$, is an admissible tracking set if $v_{C} \in \Gamma$ for all $\left(z, z_{r}\right) \in \mathbb{R}^{2} \times \mathcal{Z}_{r}$ such that $e=\left(z-z_{r}\right) \in \Lambda(\delta)$.

Proposition 1: Given positive scalars $\rho, L, C, R, \omega, V_{D C}, \quad h=1, \psi=\frac{R C}{L}$, such that $k>0$ and $R<2 \omega L$, matrix $P$ is positive definite. Moreover, the set $\Lambda(\delta)$ is an admissible tracking set if the scalar $A$ characterizing $\mathcal{Z}_{r}$ satisfies

$A \leq\left(\frac{V_{D C}}{k}-\sqrt{\frac{\delta}{(C \omega)^{2}-\left(\frac{R C}{2 L}\right)^{2}}}\right)\left(\frac{k}{k+\omega R C}\right)=: A_{r}$.

Remark 2: Following Definition 1 and Proposition 1. the largest admissible tracking set is given by the compact set $\Lambda(\bar{\delta})$, where

$\bar{\delta}:=\left((C \omega)^{2}-\left(\frac{R C}{2 L}\right)^{2}\right)\left(\frac{V_{D C}-A(\omega R C+k)}{k}\right)^{2} \geq \rho$.

It is useful to notice that $\rho$ is a given scalar that characterizes the tracking precision, while $\bar{\delta}$ depends on $A$ and $\rho$ itself, and defines the largest sublevel set of $V$ that is guaranteed to be in the basin of attraction.

\section{Control algorithm and properties of the closed-loop}

We propose a hybrid controller $\mathcal{H}_{c}=\left(\mathcal{C}, f, \mathcal{D}, G_{q}\right)$ with the objective of rendering uniformly locally asymptotically stable the set $\Lambda(\rho)$. The controller has state $q \in Q$ and input $\eta=\left(z, z_{r}\right) \in \mathbb{R}^{2} \times \mathcal{Z}_{r}$, assigned to the outputs of the plant and of the reference generator. The controller is given by the hybrid system

$$
\mathcal{H}_{c} \begin{cases}\dot{q}=0 & (q, \eta) \in \mathcal{C} \\ q^{+} \in G_{q}(\eta) & (q, \eta) \in \mathcal{D},\end{cases}
$$

where $\mathcal{C}, \mathcal{D}$, and $G_{q}$ are to be defined. In the following, we first introduce the switching logic $G_{q}$ implemented by the controller and then define the flow and jump sets $\mathcal{C}$ and $\mathcal{D}$.

We propose a jump logic which selects one among the admissible values of $q$, according to a cost function; namely, the goal is to maximize the time between consecutive switches, in order to reduce the switching frequency and the utilization of the transistors. The approach we propose to evaluate such a cost function relies on the prediction of trajectories associated to each admissible switching configuration. In the following, let $T_{P} \in \mathbb{R}_{>0}$ denote the prediction horizon.

Let $\phi \in \mathcal{S}_{\mathcal{H}}$ denote a maximal solution to the closed-loop system $\mathcal{H}=(\mathcal{C}, f, \mathcal{D}, G)$, where $\mathcal{C}, f, \mathcal{D}, G$ are to be defined. Consider $\left(t_{J}, J\right) \in \operatorname{dom} \phi$ such that $\left(q_{J}, \eta_{J}\right) \in \mathcal{D}$, where $q_{J}=q\left(t_{J}, J\right)$ and $\eta_{J}=$ $\left(z\left(t_{J}, J\right), z_{r}\left(t_{J}, J\right)\right)$. The continuous time dynamics of the predicted state $\left(\xi, \xi_{r}\right) \in \mathbb{R}^{2} \times \mathcal{Z}_{r}$ is given by

$$
\left[\begin{array}{c}
\dot{\xi} \\
\dot{\xi}_{r}
\end{array}\right]=\left[\begin{array}{c}
f_{z}(q, \xi) \\
f_{z_{r}}\left(\xi_{r}\right)
\end{array}\right], \quad\left[\begin{array}{c}
\xi(0) \\
\xi_{r}(0)
\end{array}\right]=\eta_{J},
$$

for all $q \in Q$. Then, at each jump, the proposed algorithm performs the following tasks:

1) Generate the set of admissible H-bridge configurations via the map

$$
\widehat{G}_{q}(\eta):=\left\{\begin{array}{cl}
\{q \in Q: q \leq \bar{q}\} & \text { if } e_{i}>-\frac{R C}{2 L} e_{v} \\
\{q \in Q: q \geq \bar{q}\} & \text { if } e_{i}<-\frac{R C}{2 L} e_{v} \\
Q & \text { if } e_{i}=-\frac{R C}{2 L} e_{v}
\end{array}\right.
$$

where

$$
\bar{q}:=\frac{R i_{r}-\left(L C \omega^{2}-1\right) v_{C}}{V_{D C}} .
$$

By rearranging (8), it can be shown that $\bar{q} \in$ $[-1,1]$ for all $v_{C} \in \Gamma$. With such a construction, the value of the map $\widehat{G}_{q}$ is a subset of $Q$ whose elements satisfy (9);

2) If $\widehat{G}_{q}\left(\eta_{J}\right)$ is set valued, compute the solution $t \mapsto$ $\left(\xi(t), \xi_{r}(t)\right)$ to 11 for all $\widehat{q} \in \widehat{G}_{q}\left(\eta_{J}\right)$ in a given prediction time window $\left[0, T_{P}\right]$, with $T_{P}>0$;

3) For each $\widehat{q} \in \widehat{G}_{q}\left(\eta_{J}\right)$, evaluate the time-to-impact function with respect to $\mathcal{D}$, defined by $T_{I}: Q \times$ $\mathbb{R}^{2} \times \mathcal{Z}_{r} \rightarrow \mathbb{R}_{\geq 0} \cup\{\infty\}$, where

$$
T_{I}\left(\widehat{q}, \eta_{J}\right):=\inf \left\{t>0:\left(\widehat{q}, \xi(t), \xi_{r}(t)\right) \in \mathcal{D}\right\},
$$

where $t \mapsto\left(\xi(t), \xi_{r}(t)\right)$ is the unique solution to (11) with initial condition $\eta_{J}$ and input $q$ assigned to $\widehat{q} \in \widehat{G}_{q}\left(\eta_{J}\right)$; 
4) Pick the new value $q^{+}$of $q$ according to the jump map

$$
G_{q}\left(\eta_{J}\right):=\operatorname{argmax}\left\{T_{I}\left(\widehat{q}, \eta_{J}\right): \widehat{q} \in \widehat{G}_{q}\left(\eta_{J}\right)\right\},
$$

which might be set valued.

We can now define the jump and flow set as
To prove local asymptotic stability of the closed-loop system $\mathcal{H}$ from $\Lambda(\bar{\delta})$ we follow [5, Proposition 7.5] and show that the tracking ellipse $\Lambda(\rho)$ is (strongly) forward invariant (as defined in [6]) and uniformly attractive from $\Lambda(\bar{\delta})$. As already mentioned in Section I, proofs are omitted due to space constraints and will be published elsewhere.

$$
\begin{aligned}
\mathcal{D} & :=\left\{(q, \eta) \in \mathcal{X}: \rho \leq V(e) \leq \bar{\delta}, \dot{V}(e, q, z) \geq-\lambda \frac{R}{L} V(e)\right\} \\
\mathcal{C} & :=\overline{\mathcal{X} \backslash \mathcal{D}},
\end{aligned}
$$

(e) Theorem 1: Given positive scalars $\rho, L, C, R, \omega, V_{D C}, \quad h=1, \quad \psi=\frac{R C}{L}$, such that $k>0$ and $R<2 \omega L$, the set $\Lambda(\rho)$ is uniformly locally where the parameter $\lambda \in(0,1)$ rules out the existence of multiple consecutive jumps. In particular, Lemma 1 implies that for all $v_{C} \in \Gamma$ there always exists at least one $q \in Q$ such that (9) is satisfied. Then, $\lambda<$ 1 guarantees $G(\mathcal{D}) \subset \mathcal{C} \backslash \mathcal{D}$, meaning that solutions always flow for a finite amount of time after each jump. Moreover, $\lambda>0$ ensures $\dot{V}$ is always strictly negative.

Remark 3: The presented switching logic $G_{q}$ can be applied to the general case of multilevel inverters. In such an approach, the connection of multiple inverters increases the number of degrees of freedom for the controlled input voltage. More specifically, the admissible values of the state $q$ are called "levels" and belong to the set $[-1,1]$.

When controller $\mathcal{H}_{c}$ is used as a controller for the switching system in (2), the hybrid closed-loop system with state $x:=\left(q, z, z_{r}\right) \in \mathcal{X}$ can be written as an autonomous hybrid system $\mathcal{H}=(\mathcal{C}, f, \mathcal{D}, G)$ given as

$$
\mathcal{H} \begin{cases}\dot{x}=f(x) & x \in \mathcal{C} \\ x^{+} \in G(x) & x \in \mathcal{D},\end{cases}
$$

with flow and jump maps defined as

$f(x):=\left[\begin{array}{c}0 \\ f_{z}(q, z) \\ f_{z_{r}}\left(z_{r}\right)\end{array}\right] \forall x \in \mathcal{C}, G(x):=\left[\begin{array}{c}G_{q}\left(z, z_{r}\right) \\ z \\ z_{r}\end{array}\right] \forall x \in \mathcal{D}$

We proceed with stability analysis by showing that the closed loop system $\mathcal{H}$ satisfies the hybrid basic conditions introduced in [5, Assumption 6.5].

Lemma 2: The sets $\mathcal{C}, \mathcal{D}$ and the maps $f, G$ satisfy the following:

(A1) the sets $\mathcal{C}$ and $\mathcal{D}$ are closed;

(A2) the map $f: \mathcal{C} \rightarrow \mathcal{X}$ is continuous;

(A3) the map $G: \mathcal{D} \rightrightarrows \mathcal{X}$ is outer semicontinuous and locally bounded relative to $\mathcal{D}$. Moreover, $\mathcal{D} \subset$ dom $G$.

Remark 4: Lemma 2 implies that the closed-loop system $\mathcal{H}$ satisfies the hybrid basic conditions and is therefore well-posed; see [5, Assumption 6.5]. asymptotically stable for $\mathcal{H}$ with basin of attraction containing the admissible tracking set $\Lambda(\bar{\delta})$.

Sketch of the proof: From [5, Proposition 7.5], local asymptotic stability of $\Lambda(\rho)$ is directly implied by forward invariance and uniform local attractivity of $\Lambda(\rho)$. Forward invariance comes from $G(\mathcal{D} \cap \Lambda(\rho)) \subset$ $\Lambda(\rho)$ and from the flow map $f(x)$ pointing towards the interior of $\Lambda(\rho)$ for all $x \in \partial \Lambda(\rho) \cap \mathcal{C}$, where $\partial \Lambda(\rho)$ indicates the boundary of the compact set $\Lambda(\rho)$. Local attractivity of $\Lambda(\rho)$ is guaranteed by (9) during flows and by solutions flowing for at least a finite amount of time after each jump, due to $G(\mathcal{D}) \subset \mathcal{C} \backslash \mathcal{D}$. Then, uniform local attractivity of $\Lambda(\rho)$ from the admissible tracking set $\Lambda(\bar{\delta})$ follows from $\rho>0$ and $V>0$ on the boundary of $\Lambda(\rho)$. Since the closed-loop system $\mathcal{H}$ is a well-posed hybrid system (see Lemma 2), and the attractor $\Lambda(\rho)$ is compact, [5, Theorem 7.12] implies that $\Lambda(\rho)$ is uniformly locally asymptotically stable with basin of attraction containing the admissible tracking set $\Lambda(\bar{\delta})$.

\section{Simulation Results}

In this section, we show simulation results obtained $\mathcal{D}$.with the controller proposed in this article. The closedloop system is implemented in MATLAB and simulated via the Hybrid Equations (HyEQ) Toolbox [7] 1 . The following constants are common to all the simulations: $R=1 \Omega, L=2 m H, C=1.063 m F, V_{D C}=220 \mathrm{~V}$, $\omega=120 \pi$ (or $f=60 \mathrm{~Hz}$ ), $A=100 \mathrm{~V}, \rho=16.06$, $h=1, \psi=0.5315$, and $\bar{\delta}=2241.2$.

Figure 4 shows simulations of the closed-loop system $\mathcal{H}$ with initial conditions inside the admissible tracking set $\Lambda(\bar{\delta})$. The resulting trajectories are presented in error coordinates $\left(e_{i}, e_{v}\right)=z-z_{r}$ to highlight the uniform local asymptotic stability property of the tracking ellipse guaranteed by Theorem 1 . In fact, with an initial condition inside the estimate $\Lambda(\bar{\delta})$ of the basin

\footnotetext{
${ }^{1}$ Code at https://github.com/HybridSystemsLab/HybridPredictiveInverter
} 
of attraction, solutions to $\mathcal{H}$ converge to the tracking ellipse $\Lambda(\rho)$ and are kept inside such a set.

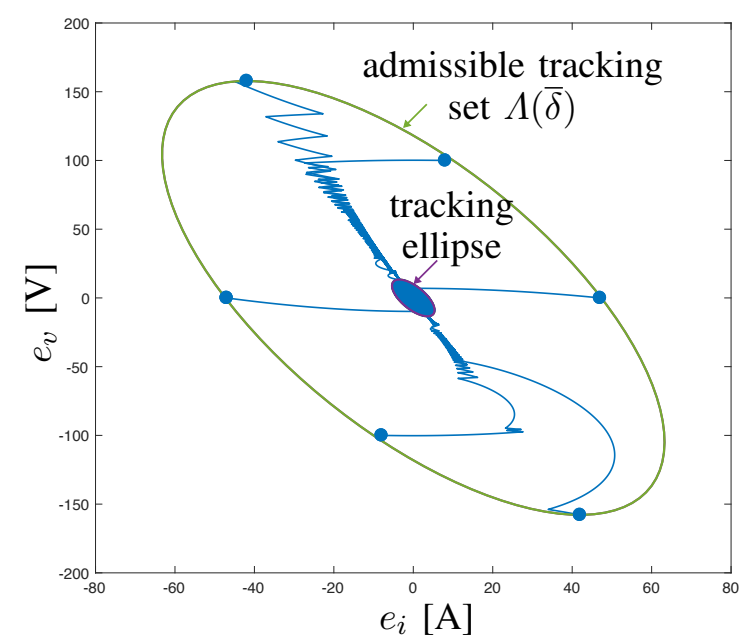

Fig. 4: Simulation of $\mathcal{H}$ with initial conditions inside the estimate $\Lambda(\bar{\delta})$ of the basin of attraction.

A validation of the robustness properties of the controller is provided in Figure 5. In this simulation, the perturbed DC input voltage $V_{D C}^{d}$ is simultaneously subjected to two noise signals:

$$
V_{D C}^{d}(t)=V_{D C}+d_{s}(t)+d_{u}(t)
$$

where

- $t \mapsto d_{s}(t) \in \mathbb{R}$ is a sinusoidal disturbance $d_{s}(t)=$ $A_{d} \sin 2 \pi f t$, with $A_{d}=2 V, f=200 H z$;

- $t \mapsto d_{u}(t) \in \mathbb{R}$ represents step changes of the form

$$
d_{u}(t)=\left\{\begin{array}{rl}
0 & t \in[0,0.1) \\
-20 & t \in[0.1,0.2) \\
20 & t \in[0.2,0.3) \\
40 & t \in[0.3,0.4) \\
0 & t \in[0.4, \infty) .
\end{array}\right.
$$

A profile plot of signal $t \mapsto V_{D C}^{d}(t)$ is shown in Figure $5 \mathrm{~b}$. An analysis of the frequency spectrum of the AC output signal is presented in Figure 6. The FFT for the signals $t \mapsto v_{C}(t)$ and $t \mapsto i_{L}(t)$ are computed for the given set of parameters and eight different initial conditions of the state $z_{r}$, with $z$ always initialized inside the tracking ellipse $\Lambda(\rho)$ centered at $z_{r}$. The fundamental harmonic of the output voltage is estimated to be at $60 \mathrm{~Hz}$, with the FFT having a frequency resolution of $0.2 \mathrm{~Hz}$. The amplitude of the output signals $v_{C}$ and $i_{L}$ at other frequencies is at least $60 \mathrm{~dB}$ and $50 \mathrm{~dB}$ smaller, respectively, than that of the fundamental component, with peaks occurring mostly at odd harmonics $(180 \mathrm{~Hz}$ and $300 \mathrm{~Hz})$. This confirms that the controller $\mathcal{H}_{c}$ allows tracking a sinusoidal signal at the given frequency $\omega$. High output power

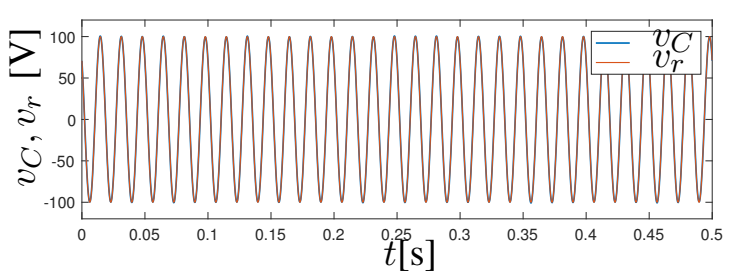

(a) Output voltage $v_{C}$ compared to $v_{r}$ in the presence of DC input disturbances.

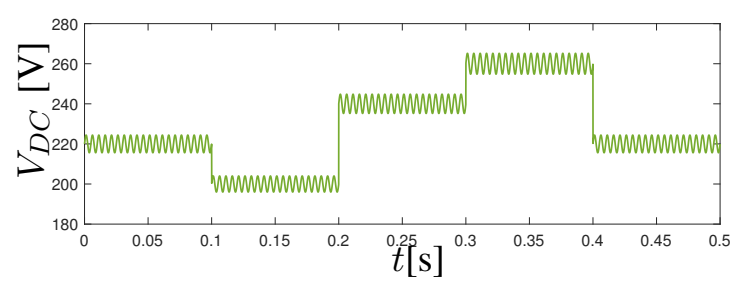

(b) Perturbed input voltage $V_{D C}^{d}$.

Fig. 5: Simulation of $\mathcal{H}$ with perturbed $V_{D C}$ input.

quality is one of the crucial characteristics required in an inverter and is typically measured in terms of Total Harmonic Distortion (THD) of the output signal. The THD of a signal $t \mapsto X(t)$ is given by

$$
\operatorname{THD}(X)=\frac{\sqrt{\sum_{n=2}^{\infty}\left(X_{n}^{2}\right)_{r m s}}}{\left(X_{1}\right)_{r m s}},
$$

where $\left(X_{n}\right)_{r m s}$ is the rms value of the $n^{\text {th }}$ harmonic of $X(t)$, while $\left(X_{1}\right)_{r m s}$ is the rms value of the fundamental frequency component. For grid-tied inverters, the IEEE standard 1547 [8] sets the maximum harmonic distortion of the output current to a value of $5.0 \%$. Simulations of the closed-loop system $\mathcal{H}$ for eight different initial conditions show that, on average, $T H D\left(v_{C}\right)=0.806 \%$ and $T H D\left(i_{L}\right)=1.001 \%$.

The benefits of adopting a prediction-based selection of $q$ can be observed in Figure 77. The closed-loop system $\mathcal{H}$ is simulated separately with and without prediction, i.e. implementing the jump maps $G$ and $\widehat{G}$, respectively. More specifically, when $\widehat{G}$ is used as switching logic, the controller merely selects $q$ among all the ones satisfying (9). Such an approach guarantees uniform local asymptotic stability of the tracking ellipse, but does not allow to select $q$ according to a specific criterion. On the other hand, when $G$ is used as switching logic in the control algorithm, $q$ is selected in order to maximize the time between consecutive switches. For the results presented in this paper, prediction of solutions is performed by an ODE solver with event detection capability. Solutions to (11) are predicted for all the admissible values of $q$ until they 


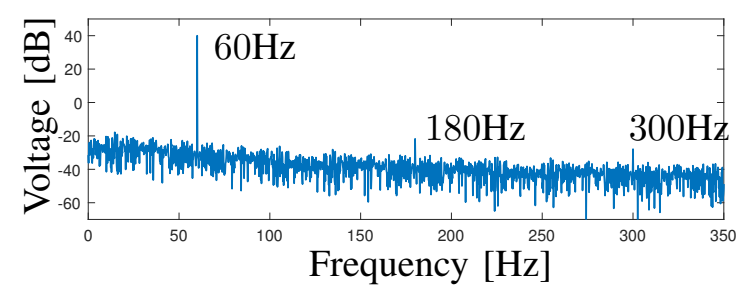

(a) FFT of $v_{C}$ averaged from eight initial conditions.

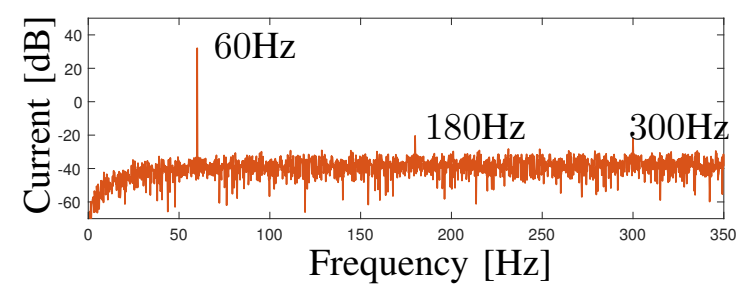

(b) FFT of $i_{L}$ averaged from eight initial conditions.

Fig. 6: Frequency analysis of the output voltage and current.

hit the jump set. Then, the $q$ minimizing the time to impact function defined in (12) is selected. It can be noticed how a prediction-based approach (Figure 7b) generates a more regular switching pattern if compared to the case without prediction (Figure 7 a), leading to a reduction of the number of switches. In this simulation, the variable $q$ experiences a $32.82 \%$ decrease - from 131 to $88-$ of the number of switches in a $20 \mathrm{~ms}$ time window.

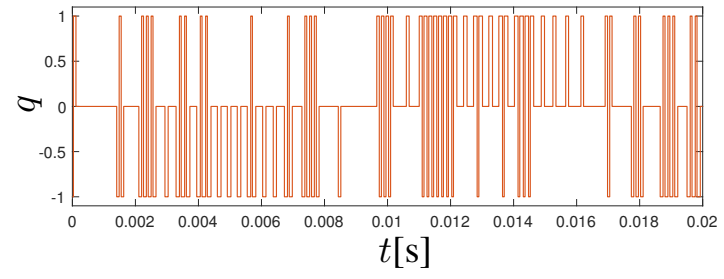

(a) Switches of $q$ without prediction.

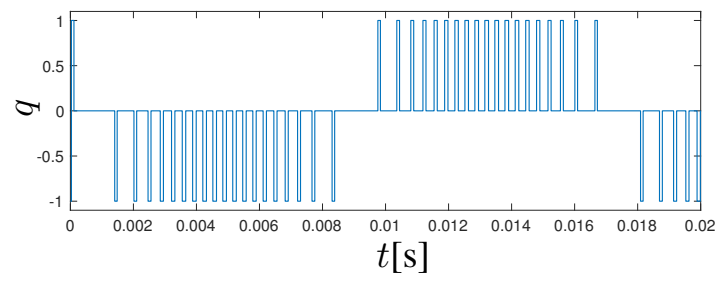

(b) Switches of $q$ with prediction.

Fig. 7: Simulation of $\mathcal{H}$ with and without a predictionbased switching logic.

Robustness of the tracking algorithm to changes in the reference signal is shown in Figure 8 . Every $50 \mathrm{~ms}$, both the amplitude and the phase of the reference are reset to a random value which satisfies the controller constraints. Disturbances in the reference signals can be associated to several scenarios, e.g. voltage or current sags due to failure of other devices connected to the grid, execution of anti-islanding policies or synchronization-related resets. As can be observed, uniform local asymptotic stability of the tracking ellipse guarantees that the output voltage $v_{C}$ converges in finite time to $v_{r}$ and remains close to it until a new reset occurs.

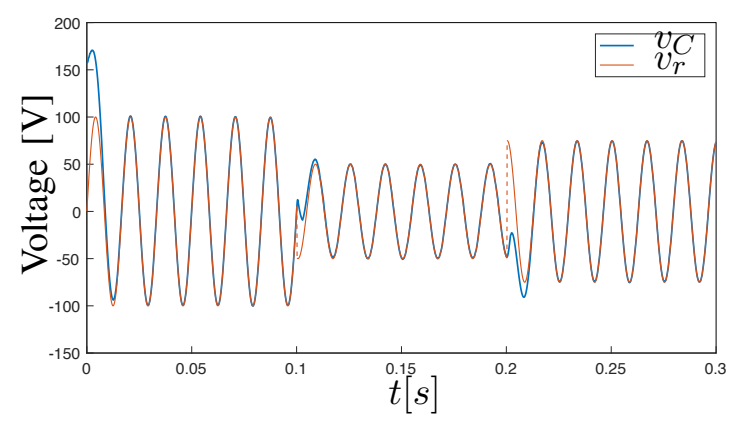

Fig. 8: Simulation of $\mathcal{H}$ with impulsive reference resets.

\section{CONCLuSions}

In this paper, we presented a hybrid predictive controller for a single-phase DC/AC inverter. The proposed algorithm guarantees tracking of a reference signal by uniformly locally converging to a forward invariant tracking ellipse around the reference trajectory, under an appropriate selection of the system parameters. Simulations show that the control algorithm is robust to impulsive changes of the reference signal, to small perturbations, and to variations of the DC input voltage, while obtaining harmonic distortion of the output signal within the standard 5\% limit for energy distribution, according to IEEE 1547. In particular, the average harmonic distortion of the simulated output voltage and current are equal to $0.806 \%$ and $1.001 \%$, respectively. We also provided simulation results illustrating the effectiveness of a prediction-based control strategy, which causes a $32.82 \%$ decrease - from 131 to 88 of the number of switches in a $20 \mathrm{~ms}$ time window.

\section{REFERENCES}

[1] N. Mohan, T. M. Undeland, and W. P. Robbins. Power Electronics. Converters, applications and design. John Wiley \& Sons, 2003.

[2] J. Holtz. Pulsewidth modulation for electronic power conversion. In Proceedings of IEEE, pages 1194-1214, 1994. 
[3] M.H. Rashid. Power Electronics Handbook. Academic, 2001.

[4] J. Chai and R.G. Sanfelice. A robust hybrid control algorithm for a single-phase DC/AC inverter with variable input voltage. In Proc. of the 2014 American Control Conference, pages 1420-1425, 2014.

[5] R. Goebel, R. G. Sanfelice, and A. R. Teel. Hybrid Dynamical Systems. Princeton University Press, 2012.

[6] J. Chai and R.G. Sanfelice. On notions and sufficient conditions for forward invariance of sets for hybrid dynamical systems. In Proc. of the IEEE Conf. on Decision and Control, pages 28692874, 2015.

[7] R. G. Sanfelice, D. A. Copp, and P. Nanez. A toolbox for simulation of hybrid systems in Matlab/Simulink: Hybrid Equations (HyEQ) Toolbox. In Proceedings of Hybrid Systems: Computation and Control Conference, pages 101-106, 2013.

[8] IEEE Standard for Interconnecting Distributed Resources With Electric Power Systems. IEEE Std. 1547-2003. 